\title{
EDITORIAL
}

\section{EL DISCURSO DE LA FLEXIBILIDAD EN EL CURRÍCULO DEL INSTITUTO DE EDUCACIÓN FÍSICA DE LA UNIVERSIDAD DE ANTIOQUIA}

\author{
THE FLEXIBILITY DISCOURSE \\ IN TO CURRICULUM OF INSTITUTO \\ DE EDUCACIÓN FÍSICA OF UNIVERSIDAD \\ DE ANTIOQUIA \\ DA FLEXIBILIDADE NO CURRÍCULO \\ DO INSTITUTO DE EDUCAÇÃO FÍSICA \\ DA UNIVERSIDADE DE ANTIOQUIA
}

\begin{abstract}
León Urrego ${ }^{1}$
1 Doctor por la Universidad de Valladolid (Espa a). Profesor Asistente del Instituto de Educaci n F sica de la Universidad de Antioquia (Colombia). E-mail: leon.urrego@udea.edu.co
\end{abstract}




\section{RESUMEN}

Este ensayo desarrolla una revisi $\mathrm{n}$ al estado y la implementaci $\mathrm{n}$ de la idea de flexibilidad en el proyecto educativo del Instituto de Educaci n F sica de la Universidad de Antioquia (Colombia). Para plantear este an lisis, se ha apelado a la revisi $\mathrm{n}$ de la documentaci $\mathrm{n}$ que la instituci $\mathrm{n}$ ofrece sobre el proceso de transformaci n, seguimiento y desarrollo curricular; adem s, se apela a la gu a te rica y conceptual planteada por D az $(2002,2011)$ y Nieto (2002). El an lisis va acompa ado de algunas ideas que permiten visualizar posibles acciones a implementar, con el prop sito de cualificar el ideal de flexibilidad en el proyecto educativo revisado.

PALABRAS CLAVE: curr culo; flexibilidad; educaci $\mathrm{n} f$ sica; transformaci $\mathrm{n}$; educaci $n$.

\section{ABSTRACT}

This essay develops a review of the state and the application of the concept of flexibility in the educational project of the Instituto de Educaci $n$ F sica PPhysical Education Institute\}, of the Universidad de Antioquia (Colombia) \{University of Antioquia\}. To present this analysis, it was necessary to review the documentation offered by the institution on the transformation process, monitoring and curriculum development; the theoretical and conceptual guide proposed by D az $(2002,2011)$ and Nieto (2002) was revised as well. The analysis is accompanied by some ideas that allow to visualize possible actions to be implemented with the purpose of qualifying the ideal of flexibility in the revised educational project.

KEYWORDS: curriculum; flexibility; physical education; transformation, education.

\section{RESUMO}

Este ensaio desenvolve uma revis o ao estado e a posta em funcionamento da ideia de flexibilidade no projeto educativo do Instituto de Educaç o F sica da Universidade de Antioquia (Col mbia). Para propor esta an lise, apelou-se revis o da documentaç o que a instituiç o oferece sobre o processo de transformaç o, rastreamento e desenvolvimento curricular; ademais, apela-se guia te rica e conceitual proposta por D az (2002, 2011) e Nieto (2002).A an lise vai acompanhada de algumas ideias que permitem visualizar poss veis aç es a executar, com o prop sito de qualificar o ideal de flexibilidade no projeto educativo revisado.

PALAVRAS-CHAVE: curr culo; flexibilidade; Educaç o F sica; transformaç O; educaç O 


\section{LA DIMENSIÓN DE LA FLEXIBILIDAD EN EL INSTITUTO DE EDUCACIÓN FÍSICA DE LA UNIVERSIDAD DE ANTIOQUIA}

La noci $\mathrm{n}$ de flexibilidad en el contexto de la formaci $\mathrm{n}$ universitaria encuentra su escenario de divulgaci $n$ e implementaci $n$ en la pol tica curricular que ha guiado en la d cada anterior a las instituciones educativas colombianas. El Instituto de Educaci $n$ F sica de la Universidad de Antioquia (Colombia) (en adelante Instituto) ha asumido en su dise o curricular (IUEF, 2005) el discurso relacionado con la flexibilidad de una manera limitada. M s adelante se tratar de mostrar c mo se dispone de una porci $n$ reducida y b sica de esta noci $n$, que admite mayores implicaciones.

La documentaci $\mathrm{n}$ institucional que plasma la pol tica curricular, contempla la flexibilidad como una caracter stica metodol gica que permite concretar los principios pedag gicos ${ }^{1}$ del proyecto educativo del Instituto. Otras caracter sticas que aparecen en dichos textos son: conexi $n$ e interacci $n$, privilegio a los procesos, integraci $n$ de teor a y pr ctica, evaluaci $n$ permanente y colectiva, participaci $n$, apertura, contextualizaci n socioecon mica y cultural.

Tales caracter sticas metodol gicas del proyecto educativo se inscriben en una ideolog a que asume el discurso sobre la flexibilidad en funci $n$ de una propuesta de innovaci $n$ y transformaci $n$ curricular originada en el Instituto a inicios de la $d$ cada de 1990. Este proceso de cambio se moviliz a partir de cuestionar los fundamentos acad micos que tradicionalmente ven an orientando la formaci $\mathrm{n}$ de los profesionales licenciados en educaci $\mathrm{n}$ f sica; se procur integrar a los actores para "reflexionar, proponer y concertar" una propuesta de transforma-

1 Para el caso del Instituto son: reflexi $\mathrm{n}, \mathrm{m}$ Itiples perspectivas, interacci n comunicativa, transformaci n, autodirecci n, di logo cultural. 
ci n basada en la construcci n "participada, abierta y flexible" (IUEF, 2005, p.3).

Se visualiza la presencia de la noci $\mathrm{n}$ de flexibilidad en las bases de la transformaci n curricular del Instituto. El proceso de cambio eligi el modelo pedag gico constructivista como ideolog a formativa para guiar el proyecto educativo, y entre todas las argumentaciones presentadas para sustentar esta elecci $n$, nuevamente aparece la "construcci n permanente, flexible y participada de la comunidad educativa" (IUEF, 2005, p.6).

La Gu a del dise o curricular, documento $\mathrm{p}$ blico empleado para ilustrar a los profesores noveles, ocasionales y de c tedra sobre el proyecto educativo del Instituto, menciona la noci $\mathrm{n}$ de flexibilidad de manera indeterminada, inconsistente e imprecisa. Sin embargo, esta gu a, en su apartado sobre la metodolog a curricular, detalla la flexibilidad como caracter stica del proyecto educativo en los siguientes $t$ rminos:

[...] implica apertura y autodirecci $\mathrm{n}$ respecto a la formulaci $\mathrm{n}$ y b squeda de metas, a la selecci $\mathrm{n}$ de materiales did cticos, a la escogencia de pr cticas y al dise o de los ambientes donde ha de tener lugar el aprendizaje. Igualmente demanda adecuaci $n$ de contenidos desde la visi n conjunta de los implicados en el acto de aprender, donde el respeto a la diferencia y el di logo de saberes se ale el camino para la autodeterminaci $n$ personal y social, siempre con miras a la formaci $\mathrm{n}$ de una conciencia $\mathrm{cr}$ tica y transformadora (IUEF, 2005, p.15).

Estas son las nicas rese as directas en la Gu a Curricular del Instituto al principio de flexibilidad. En el p rrafo citado se interpreta que las ideas aluden a diversos aspectos indiferenciados que no ofrecen claridad $y$, de este modo, su concreci n o realizaci $n$ queda en un plano abstracto. Es decir, se mezclan aspectos referidos al profesorado, a los estudiantes y a los gestores curriculares, que posiblemente guardan coherencia con el modelo educativo (constructivista, donde los diferentes acto- 
res participan activamente de modo cooperativo) que orienta el proyecto curricular, pero que, a la vez, pueden propiciar confusi $n$ o indefinici $n$ con respecto a sus alcances, posibilidades y imitaciones.

El $p$ rrafo citado tambi $n$ permite interpretar que se asume la flexibilidad en un sentido positivo, en una perspectiva de formaci $\mathrm{n}$ producto de la evoluci $\mathrm{n}$ de los modelos educativos, o $\mathrm{s}$ lo como una cuesti $\mathrm{n}$ dada para mejorar el proyecto educativo, sin mayores datos sobre sus alcances y consecuencias. Simplemente se resaltan las bondades, pero poco o nada se indica sobre el compromiso institucional con respecto a los recursos, a los aspectos ideol gicos, o a las adaptaciones contextuales que exige la materializaci $\mathrm{n}$ del discurso de la flexibilidad para el Instituto.

En concreto, la pol tica curricular del Instituto admite una gran diversidad de asuntos con relaci $\mathrm{n}$ a la flexibilidad, pero no ofrece claridad sobre los alcances e implicaciones de asumir este discurso en su proyecto educativo. Se puede leer una descripci n superficial, que adem s ofrece estrategias y acciones de concreci $\mathrm{n}$ bastante someras en el proyecto educativo. Sin embargo la presencia de esta noci $\mathrm{n}$ en el discurso tiene por utilidad mostrar dinamismo, innovaci n, pertinencia o, mejor, un nuevo enfoque curricular.

Esta situaci $\mathrm{n}$ parece guardar proximidad con el fen meno de globalizaci n y neoliberalismo que ha ido posicion ndose (posesion ndose) en (de) los proyectos educativos. Aqu, vale mencionar a Harvey, quien se ala que la forma discursiva hegem nica "posee penetrantes efectos en los modos de pensamiento, hasta el punto de que ha llegado a incorporarse a la forma natural en que muchos de nosotros interpretamos, vivimos y entendemos el mundo" (2006, p.7).

Como consecuencia, quedan sin precisi $n$ o determinaci $n$ los detalles que aluden a los espacios y estrategias de realizaci $n$ o concreci $n$ de dichas ideas, aquello que involucra y las 
consecuencias que se despliegan con un enfoque curricular que "admite" la flexibilidad. De todo esto resulta importante que el Instituto redimensione, aclare y precise aquello que asume en su proyecto curricular, con la ideolog a que quiere implementar; que detalle las acciones y estrategias que se precisan para concretar el discurso de la flexibilidad, contextualizado a las situaciones particulares del Instituto.

\section{EL DISCURSO DE LA FLEXIBILIDAD Y SU PERSPECTIVA CRÍTICA}

Al inicio de este escrito se afirm que la noci $n$ de flexibilidad es mucho $\mathrm{m}$ s amplia y compleja que la que se asume y se busca implementar en el Instituto. Corresponde mostrar los I mites y dimensiones que ampl an la comprensi $n$ de este $t$ rmino, para lo cual nos apoyaremos en los trabajos del colombiano Mario D az Villa (D az, 2002, 2011) y de la mexicana Luz Nieto Caraveo (Nieto, 2002). El inter s ac es revisar el estado de este principio contempor neo de transformaci $n$ educativa en el Instituto, y aportar algunas ideas que permitan avanzar en el despliegue de todo el potencial que podr an tener los discursos acerca de la flexibilidad.

D az $(2002,2011)$ ha desarrollado en la Itima d cada un importante trabajo acad mico e investigativo acerca de la flexibilidad en el contexto educativo colombiano, y ha asesorado procesos curriculares en Per y $\mathrm{M}$ xico. Como consecuencia de esto, ha logrado desarrollar investigaciones conjuntas con Nieto (2002), quien se ha ocupado de reflexionar sobre la flexibilidad educativa en la Educaci n Agron mica.

Las ideas $\mathrm{m}$ s gen ricas sobre la noci $\mathrm{n}$ de flexibilidad las ofrece $\mathrm{D}$ az, quien se ala que se trata de una forma de discurso asociada al neoliberalismo, a la globalizaci $\mathrm{n}$ y a sus derivaciones culturales, denominadas por los expertos como postmodernidad 
(D az, 2011, p.10). Este discurso, que ha trascendido a la educaci $n$, constituye una forma de globalizaci $n$ de un tipo de cultura que busca la apertura, el descentramiento, la desterritorializaci $n$, la desclasificaci $n$ y la integraci $n$; una cultura que reside en los esquemas econ micos y de mercado, otros modos de organizaci $\mathrm{n}$ de la producci n, migraciones masivas, movimientos en las formas de producci $n$ y difusi $n$ del conocimiento, acceso a la informaci $n$ y m Itiples fuentes de cultura, y transformaciones profundas de la vida cotidiana y de la identidad de los sujetos.

De los anteriores aspectos, D az resalta con mayor nfasis aquellos que determinan el "mundo laboral", porque considera que han generado consecuencias para los procesos de formaci $n$ que se han visto mediados o absorbidos por las I gicas de los mercados; las instituciones se (las) han movilizado a la creaci $\mathrm{n}$ de condiciones de cambio hacia esta I gica, que incluyen modificaciones pedag gicas, evaluativas y curriculares.

El cambio cultural, seg $\mathrm{n}$ el autor, tiene por origen el ajuste de modelo econ mico, donde se pasa de una forma de producci $n$ masiva y $r$ gida, a un modo din mico de producci $n$ limitado pero adaptado a las nuevas econom as que no demandan cantidad, sino contextualizaci n, flexibilidad, adaptaci n y valor a adido a partir de la subjetividad. En resumen, un modelo "competente", lo que se ha dado en llamar "nueva econom a de la producci n y mercado flexible" (D az, 2011, p.15).

El autor ubica el parentesco de la I gica de la globalizaci $n$ con la "especializaci $n$ flexible", porque esta idea de formaci $n$ conviene a la alta tecnolog a, a la velocidad, al acceso inmediato a los mercados globales; en s ntesis, la flexibilidad es la $v$ a para atender las demandas de los tiempos que corren (D az, 2011, p.8). Frente a esta I gica predominante, considera que el gran problema para las instituciones educativas es que han asumido estos discursos y pr cticas sin "mayores resistencias", de una 
forma instrumental, y se han puesto al servicio de la I gica de formaci $n$ profesional flexible (D az, 2011, p.18).

Las consecuencias de asumir la flexibilidad en la formaci $n$ son insospechadas, pero se sabe que su relaci $\mathrm{n}$ con el sistema econ mico admite las condiciones "laborales precarias, su producci n 'justo a tiempo', sus mercanc as informacionales", que al mismo tiempo contrastan con las im genes de generaci $\mathrm{n}$ de "espontaneidad, creatividad, cooperaci n, movilidad, relaciones entre iguales, aprecio por la diferencia, apertura a experimentar el presente" (D az, 2011, p.19).

As las cosas, la flexibilidad no es un mero dispositivo ret rico del discurso curricular, sino $\mathrm{m}$ s bien la materializaci $\mathrm{n}$ y expresi $\mathrm{n}$ de la cultura contempor nea, y se convierte en un reto para las instituciones educativas asumirse en esta I gica, considerando las posiciones pasivas e instrumentales, o asumiendo una din mica cr tica que permita desplegar la forma $\mathrm{m} s$ favorable a los sujetos y a la cultura, caracterizada hoy por demandar opcionalidad, pluralidad, contingencia, circunstancialidad.

Lo planteado hasta ac permite dimensionar las ideas $\mathrm{m} \mathrm{s}$ gen ricas sobre la noci $\mathrm{n}$ de flexibilidad. Ahora se a adir $\mathrm{n}$ argumentos, buscando precisar las formas y lo que involucra la flexibilidad en las instituciones educativas, y se revisar $\mathrm{n}$ algunos elementos de la gu a curricular del Instituto, para analizar el nivel de asimilaci n que en dicho documento se hace de la flexibilidad.

Tomaremos como base ideal la definici n que aporta Nieto, quien se ala que una de las implicaciones que traer a la flexibilidad en el contexto educativo en toda su amplitud, ser a el debilitamiento de los principios de clasificaci $n$ y enmarcamiento con que opera la educaci n: "significa reconstruir los mbitos de libertad, responsabilidad, confianza y autonom a con que funcionamos. En s ntesis significa modificar la distribuci $\mathrm{n}$ y formas de ejercicio del poder en nuestras instituciones" (2002, p.7).

Nieto menciona tres perspectivas de la flexibilidad: instrumental, pol tica y te rico conceptual. La instrumental alude a 
los modos y formas como se concreta la flexibilidad; la pol tica se refiere a los factores de distribuci $\mathrm{n}$ de poder y control de la instituci $n$; y la te rico conceptual acoge las argumentaciones sobre el contexto hist rico, las tendencias y corrientes, sus prop sitos, los mbitos de aplicaci n, las modalidades, los alcances y limitaciones del discurso de la flexibilidad (2002, p.8).

De conformidad con estas tres perspectivas de la flexibilidad, el discurso del proyecto educativo del Instituto podr a caracterizarse por presentar rasgos que lo identificar an con la perspectiva instrumental, pues la Gu a Curricular, en su caracterizaci $\mathrm{n}$ metodol gica, asocia flexibilidad con auto-direcci $\mathrm{n}$, formulaci $n$ y b squeda de metas, selecci $n$ de materiales, selecci $\mathrm{n}$ de pr cticas y dise o de ambientes de aprendizaje, aunque, como se ha planteado, ofrece poca informaci $n$ acerca de c mo y por qu se logr determinar que estas manifestaciones de la flexibilidad ser an las $\mathrm{m}$ s apropiadas y adecuadas a la realidad institucional.

Al lado de lo anterior, el proyecto curricular, en su dimensi $n$ flexibilidad, tambi $n$ presenta rasgos en perspectiva pol tica. Nieto (2002) se ala que este tipo de aspectos generalmente permanecen ocultos o invisibles, al tratarse de elementos ideol gicos inmanentes que permean todas las esferas de los proyectos educativos, culturales y sociales. Tambi $n$ es evidente que la Gu a Curricular objeto de an lisis, admite que el modelo pedag gico es el constructivista, y en la totalidad del documento presenta elementos que lo erigen como la ideolog a predominante. Desde este punto de vista, Nieto se ala que, en ocasiones, los modelos curriculares o pedag gicos "idealizados" que no atienden suficientemente, o con claridad, los factores de la perspectiva pol tica, suelen quedarse a nivel de "ret rica, justificando simulaciones" (2002, p.9).

En las discusiones del comit encargado de la implementaci $n$, control, evaluaci $n$ y transformaci $n$ del curr culo en el Instituto, se tratan los problemas para llevar a cabo el "cambio 
curricular", entre los cuales se destaca la dificultad de algunos actores curriculares para la asimilaci $\mathrm{n}$ y adopci $\mathrm{n}$ de la ideolo$\mathrm{g}$ a que gu a el proyecto educativo. Esta situaci $\mathrm{n}$ se puede ver en el reporte que analiza las limitaciones en la instituci $n$ para realizar la transformaci $n$, admitiendo que el proceso de transformaci n curricular presenta algunos problemas, entre ellos "la resistencia al cambio por parte de algunos docentes" (Casta eda et al., 2010, p.5).

La Itima perspectiva que se ala Nieto, indica que las teo$r$ as y concepciones sobre flexibilidad son importantes porque permiten ampliar la mirada y comprender mejor las caracter sticas que deben reunir las propuestas curriculares (2002, p.9). Al respecto, el proyecto curricular es bastante ambiguo, al punto que, en el reporte citado, se destaca el siguiente apartado:

[La] flexibilidad curricular ha creado tensi $\mathrm{n}$ debido a que muchos de los aspectos tenidos en cuenta por este principio no han sido asimilados de manera pertinente por la comunidad, esto ha llevado a concentrar la atenci n nuevamente en asignaturas o disciplinas delimitadas y aisladas, lo cual va en contraposici n con el desarrollo actual del conocimiento y con el modelo proyectado por el Instituto (Gallo \& Casta eda, 2011, p.13).

Los comentarios del comit encargado del seguimiento curricular sobre la flexibilidad, permiten formular interrogantes sobre la "asimilaci $n^{\prime}$ de esta noci $n$ en el proyecto educativo del Instituto. Teniendo en cuenta que la transformaci n curricular se propicia por la construcci n permanente, flexible y participada de la comunidad educativa, no son claras las causas por la cuales algunos actores educativos no "asimilan" la ideolog a. Tanto D az (2002) como Nieto (2002) indican que, en ocasiones, el discurso de la flexibilidad se emplea en el contexto educativo en un modo ret rico, que imprime la sensaci $\mathrm{n}$ de cambio, transformaci n o actualidad, por lo que resulta posible que el diagn stico realizado por el comit curricular del Instituto est reflejando este modo discursivo en la instituci n. 
Frente a esta situaci n, convendr a precisar, puntualizar y reorganizar el proyecto educativo en torno a cuatro mbitos de la flexibilidad abordados por ambos autores: acad mico, pedag gico, administrativo y curricular (D az, 2002; Nieto, 2002). Estos mbitos permitir an encauzar, de una manera $\mathrm{m} \mathrm{s}$ sistem tica, las transformaciones curriculares que implican el principio de flexibilidad. A continuaci $n$, se presentan las ideas que explican cada mbito, y se analiza la situaci $\mathrm{n}$ del Instituto con respecto a cada uno.

\section{FLEXIBILIDAD ACADÉMICA}

En este mbito, los autores clasifican las formas de organizaci n, relaci $n$, comunicaci $n$ y coordinaci $n$ entre los conocimientos que participan en la formaci $n$, lo que se materializa en las nominaciones organizativas, unidades acad micas y jerarqu as funcionales de las instituciones educativas, estructuras, formas organizativas o administrativas dise adas tanto para la producci n, como para la reproducci $n$ del conocimiento. Las figuras que ejemplifican este mbito de la flexibilidad en el contexto de la universidad colombiana son las Facultades, las Escuelas, los Institutos y los Departamentos.

En el caso del Instituto, se reconoce que presenta un nivel de independencia acad mica con respecto a otras unidades, facultades, escuelas o institutos, que le permite direccionar el conocimiento que tramita, pero tambi $\mathrm{n}$ se ha aislado, al punto de no presentar interdependencias funcionales con otras unidades acad micas; solo espor dicamente hay integraci n, articulaci n y complementariedad en investigaci $n$ y extensi $n$ (servicios). El proyecto curricular del Instituto no contempla prospectivamente su papel inter-disciplinario.

En este sentido, la flexibilidad acad mica est restringida por el nivel de aislamiento con respecto a otras unidades acad micas de la universidad, especialmente en el caso de la docencia. 
Esta situaci $\mathrm{n}$ alimenta un sistema acad mico est tico y $\mathrm{r}$ gido, porque el aislamiento y la desarticulaci $\mathrm{n}$ genera, seg $\mathrm{n} \mathrm{D}$ az, la configuraci $\mathrm{n}$ y el "mantenimiento de las fronteras $\mathrm{r}$ gidas tanto entre las unidades acad micas como entre los campos de conocimiento" ( 2002, p.102).

Sin duda, para el Instituto ser a importante retomar algunos aspectos formativos (contenidos, investigaciones, asignaturas, servicios) en conjunto con otras unidades acad micas, pues la forma de organizaci $\mathrm{n}$ acad mica que ahora predomina favorece el aislamiento, margina de la I gica contempor nea de producci $\mathrm{n}$ de conocimiento y obstaculiza formas de relaci $\mathrm{n}$ que podr an generar innovaci n. Sin embargo, algunos actores educativos sostienen que esta din mica otorga autonom a $y$, por tanto, los niveles de desarrollo acad mico disciplinar se ven fortalecidos.

D az (2002, p.103) tambi n se ala que la forma contempo$r$ nea de organizaci $n$ acad mica de las universidades presenta m Itiples problemas, entre los cuales se destacan: la tendencia al monopolio de los profesores de cada unidad acad mica alrededor de un rea de conocimiento espec fica, con la exclusi $n$ de aquellos que pertenecen a otras unidades; la monofuncionalidad del rol de docencia, en detrimento de la investigaci n, y viceversa; la reducida comunicaci $n$ y movilidad de profesores entre unidades acad micas; la duplicaci $\mathrm{n}$ de contenidos y responsabilidades dentro de las diferentes unidades acad micas, y dentro de la propia; la tendencia al aislamiento de los profesores; la reducci $n$ en la capacidad autocr tica; y la jerarquizaci $n$ entre reas y disciplinas de conocimiento.

Con respecto a estos problemas, el Instituto est en mora de realizar una valoraci $\mathrm{n}$ de su nivel de apertura, autonom a, interdisciplinariedad e interfuncionalidad, en el contexto de la producci $\mathrm{n}$ de conocimiento y de la misma Universidad. Esta valoraci $\mathrm{n}$ podr a evidenciar la necesidad de reorganizar las relaciones con otras unidades acad micas de la Universidad de 
Antioquia; incrementar las actividades de investigaci $n$ y los servicios de extensi $n$, evitando duplicar actuaciones y uso de recursos en los cuales podr an retroalimentarse varias unidades acad micas; fortalecer las relaciones entre profesores en t rminos de mayor comunicaci n, colaboraci $n$ y movilidad; innovar en conocimientos compartidos que podr an materializar investigaci $n$ y formaci $n$; articular programas y contenidos de uno o varios programas hacia la interdisciplinariedad; y potencializar la cr tica y la autocr tica, sometiendo a constante revisi $\mathrm{n}$ las jerarqu as de la estructura acad mica.

Acciones como estas podr an conducir a concretar el ideal de la flexibilidad acad mica, pues es la apertura a formas de organizaci $n$ y relaci $n$ lo que fortalece la interacci $n$ de conocimientos, saberes y disciplinas, la interacci $n$ entre investigaci $n$, docencia y extensi $n y$, en Itimas, la interacci $n$ de actores educativos.

\section{FLEXIBILIDAD PEDAGÓGICA}

Es propio del discurso contempor neo sobre educaci n, idealizar los modelos pedag gicos flexibles en la relaci $n$ ense anza-aprendizaje, y las formas en que esta se asume, el grado de plasticidad y diversidad que admite. Para Nieto (2002, p.10), en este principio de flexibilidad operan las formas como se organizan los aprendizajes, los roles que asumen profesores y estudiantes, los apoyos con los que se cuenta, las formas de evaluaci $n$ y calificaci $n$, los materiales, los medios y los espacios disponibles para desarrollar los procesos de aprendizaje.

Por su parte D az (2002, p.106) se ala que ac est comprometida la libertad y el control que puede tener el estudiante sobre su propio aprendizaje, lo que implica la personalizaci $n$ de las competencias demandadas por los contextos. Tambi $n$ hace hincapi en la relaci $\mathrm{n}$ estudiante-profesor, donde nece- 
sariamente debe haber un cambio en la I gica de transmisi $n$ de conocimiento por la disposici $\mathrm{n}$ a los procesos de aprendizaje y construcci $\mathrm{n}$ de conocimiento.

A este respecto, el Instituto ha procurado desplegar e implementar un modelo educativo cr tico y pr ctico que busca tensionar las relaciones maestro-estudiante, admitiendo a estos Itimos como sujetos complejos, a quienes no tiene sentido ofrecer una formaci $n$ homog nea, sino que esta var a seg $n$ los contextos formativos y los estudiantes dan sentido e intencionalidad a sus aprendizajes. Sin embargo, los procesos de acreditaci $n$ han generado revisiones con respecto al modelo educativo del Instituto; en el documento del comit de curr culo se indica que el modelo se someti a revisi $\mathrm{n}$ bajo la asesor a de expertos, lo cual tambi $\mathrm{n}$ incide en la concreci $\mathrm{n}$ de la flexibilidad.

A partir de la lectura de D az (2002) y Nieto (2002), los dos autores gu a con respecto a la flexibilidad, se sugieren algunas ideas para la revisi $\mathrm{n}$ que actualmente se adelanta en el Instituto: en el proceso de ajuste que adelanta el comit curricular, se podr a pensar el modelo educativo en funci n del aprendizaje, de las relaciones sociales con los contextos profesionales, del despliegue de pr cticas en escenarios distintos a los tradicionales, buscando nuevos significados e interacciones para (re) producir otras formas de conocimiento. Ello exige una disposici $\mathrm{n}$ de la instituci $\mathrm{n}$ a relaciones abiertas, flexibles e interdependientes por parte de los actores del proyecto educativo.

\section{FLEXIBILIDAD ADMINISTRATIVA}

Esta hace referencia a los ordenamientos y las relaciones de poder que se establecen en las instituciones educativas, a las formas de comunicaci $\mathrm{n}$ entre los actores, a los niveles de jerarqu a $y$, en este sentido, "puede tener impacto sobre los ordenamientos de los profesores, de las unidades acad micas" 
(D az, 2002, p.110). Este conjunto de aspectos se refiere a los procedimientos, normas y estilos de planeaci $n$, evaluaci $n$ y gesti n. Comprende las interacciones, tanto en el interior de la instituci $\mathrm{n}$ entre los actores y las sub-unidades, como en el exterior con las dem s instituciones. El discurso ideal sobre la flexibilidad administrativa abarca un sistema de relaciones abierto a los actores educativos y a las sub-unidades, pero adem s con las instituciones externas involucradas en el proyecto educativo.

Las pol ticas de interacciones institucionales e interinstitucionales en el Instituto siguen las directrices generales de la Universidad, y en gran medida su avance y desarrollo ha variado en funci $\mathrm{n}$ de la din mica que cada administrador ha querido imprimir a este modo de flexibilidad. Se requiere entonces una idea prospectiva institucional de la flexibilidad administrativa, que permita afianzar las relaciones intra e interinstitucionales de cara a garantizar este principio del cambio curricular, y de este modo se podr a dar un giro a la I gica t cnica-operativa de la administraci $n$, a un proceso que tenga alcances de tipo cultural, donde se visualice a largo plazo la importancia que tiene para la instituci $\mathrm{n}$ la gesti $\mathrm{n}$ de las relaciones con una perspectiva flexible.

Siguiendo a D az (2002, p.110), en este giro hay que tener en cuenta que cada vez mas circulan diferentes tipos de estudiantes, se formulan diversas demandas de cada vez mas variados sectores de trabajo, desempe o y producci $n$ a las profesiones, y que el desarrollo de conocimiento implica una din mica que excede la capacidad institucional, y por tanto se requieren apoyos e interacciones con distintas instituciones.

Nuevamente analizando el caso del Instituto, se puede decir que las reformas administrativas han estado casi inm viles en la Itima d cada, conservando la misma estructura administrativa de gesti $n$ curricular con la que fue planteada la reforma curricular de inicios de los a os noventa. A este respecto, es preciso imprimir estrategias din micas que permitan trascender en la fle- 
xibilidad administrativa. Por ejemplo, es importante promover la emergencia constante de grupos de estudio, investigaci $\mathrm{n}$ y trabajo, que eviten a toda costa la creciente jerarquizaci n y estancamiento de las I gicas que imprimen los grupos hegem nicos en el Instituto. En tal sentido, cabe citar de nuevo a D az, quien advierte que, para "que la flexibilidad tenga xito se requieren reformas en el dise o organizativo y el desarrollo de enfoques antiburocr ticos y descentralizadores que permitan fortalecer las capacidades innovadoras de los grupos acad micos y administrativos" (2002, p.110).

Tambi n podr a servir, en el prop sito de imprimir din mica, la aplicaci $\mathrm{n}$ de nuevos valores administrativos, donde no solo se posibiliten los recursos a los grupos (investigaci n, estudio, trabajo) tradicionales, sino que se apoye la emergencia de nuevas propuestas. Adem s, algo en lo que el Instituto a n no se ha cuestionado $\mathrm{cr}$ ticamente es en sus relaciones con las instituciones externas a la Universidad, algo que permitir a explorar cruces y trabajos integrados y combinados, contrario a lo que se presenta hoy, donde la relaci $\mathrm{n}$ se limita a la prestaci $\mathrm{n}$ de servicios/conocimientos (universidad) a usuarios (actores externos).

\section{FLEXIBILIDAD CURRICULAR}

Se trata de la adaptaci $\mathrm{n}$ a los ritmos, estilos, valores culturales, competencias, intereses y demandas que la sociedad y el contexto plantean a la instituci $n$ en un momento determinado. Para su implementaci $\mathrm{n}$ en las instituciones de educaci $\mathrm{n}$ se requiere la revisi $\mathrm{n}$ de discursos, pr cticas y estructuras organizativas tradicionales, procurando atender a los intereses y necesidades de los estudiantes y sus entornos sociales. Los autores que sirven como referente en este ensayo, relacionan la flexibilidad curricular con las estructuras, reas, asignaturas, componentes, ciclos o n cleos curriculares, entre otros (D az, 2002; Nieto, 2002). 
Para D az (2002) se trata de la distribuci n y organizaci $n$ del curr culo, que parte de la selecci $\mathrm{n}$ de contenidos (de acuerdo con su pertinencia, impacto, efectos, utilidad), el dise-

o de una forma que permite configurar especializaciones, y a la vez permite atender grandes espacios en los que se genera la producci n de conocimiento espec fico, la demarcaci $\mathrm{n}$ de reas propias y ajenas a los conocimientos, el establecimiento de secuencias que concretan las especificidades disciplinarias y cient ficas. Estos elementos componen un tipo de identidad que var a en cada curr culo de acuerdo con su contexto, y a la flexibilidad que tengan sus formas organizativas para adaptarse a las situaciones sociales, espaciales y temporales en las que se despliega.

En el caso del Instituto, la flexibilidad curricular se ha implementado de forma parcial, pues el comit curricular ha diagnosticado que el cambio en la forma organizativa del proyecto educativo solo "ha sido implementado por algunos docentes en el desarrollo de los ejes"; esta aplicaci n parcial basta para proyectar otro tipo de formaci $\mathrm{n}$ que redimensiona la "raz $\mathrm{n}$ de ser del licenciado en la educaci $\mathrm{n}$ formal y la educaci $\mathrm{n}$ para el desarrollo humano y el trabajo". El documento de evaluaci n y seguimiento del curr culo no contempla acciones o estrategias para promover la apropiaci $\mathrm{n}$ de este tipo de flexibilidad por parte de algunos actores, aunque s se ala sus efectos: "ha llevado a estudiantes y a profesores a $\mathrm{m}$ Itiples interpretaciones que en ocasiones desvirt a aspectos esenciales" (Casta eda et al., 2010, p.13).

La implementaci $\mathrm{n}$ de la transformaci $\mathrm{n}$ curricular supuso el establecimiento de dos grandes ejes transversales en la formaci $n$ profesional de licenciados en educaci $n \mathrm{f}$ sica: la investigaci $n$ y la pr ctica pedag gica, que en principio deber an estar articulados o integrarse. Esta estrategia no ha sido acogida completamente: solo algunos docentes se han comprometido 
debido a cuestiones administrativas, profesionales y disciplinares. Esto porque no se han dispuesto los recursos necesarios (que no son exclusivamente econ micos) para la aplicaci $\mathrm{n}$ de dicho plan; algunos actores se resisten al cambio y a su mayor compromiso con procesos formativos que les demandan, por ejemplo, la competencia investigativa. Por otro lado, porque a $\mathrm{n}$ persisten con fuerza mitos profesionales que desvirt an la investigaci $n$ frente a los procesos de pr ctica e intervenci $n$ pedag gica del licenciado. Y, para completar este panorama complejo de flexibilidad curricular, algo que a $\mathrm{n}$ es $\mathrm{m} \mathrm{s}$ cr tico es que algunos de quienes se han atrevido a desplegar la investigaci $\mathrm{n}$ como componente formativo, la consideran aislada y desarticulada de las "actuaciones pr cticas". Existe pues una fuerte y marcada separaci $n$ en el escenario formativo entre la pr ctica y la teor a.

Esta realidad se aleja de las visiones contempor neas, que consideran que la producci $n$ de conocimientos se ve enriquecida y fortalecida cuando se asume que las formas tecnol gicas de la vida no tienen distancia entre conocimiento y pr ctica, y que para las demandas actuales se requiere la "opcionalidad", que sustituye la rutina de hacer siempre las mismas cosas. Las formas contempor neas implican la contingencia de las circunstancias, y la mejor opci $\mathrm{n}$ para atenderlas es asumi ndolas desde la investigaci $n(D$ az, 2011, p.21) .

\section{REFERENCIAS}

1. Casta eda, G., Castro, J., Aguilar, R., L pez, N., Jim nez, J., \& Ochoa, V. (2010). Tensiones internas y externas que han incidido en los procesos de transformaci $n$ curricular en el instituto de Educaci n F sica en la Universidad de Antioquia. Medell n: Universidad de Antioquia, Instituto Universitario de Educaci n F sica, Comit de Curr culo.

2. D az, M. (2002). Flexibilidad y educaci $n$ superior en Colombia (Vol. 2). Bogot : ICFES. 
3. D az, M. (2011). Los discursos sobre la flexibilidad y las competencias en educaci n superior. Pedagog a y Saberes, 35, 9-24.

4. Gallo, L., \& Casta eda, G. (2011). La motricidad en el campo de la formaci $n$ en los programas del Instituto de Educaci n F sica (Documento in dito). Medell n: Universidad de Antioquia, Instituto Universitario de Educaci n F sica.

5. Gracia, . (2000). Conceptualizaci n, objeto de estudio, evoluci $n$ hist rica y cuerpo de conocimiento de las ciencias del deporte. $L$ dica Pedag gica, 1(5), 1-9.

6. Harvey, D. (2006). Breve historia del neoliberalismo. Espa a: Ediciones Akal.

7. IUEF Instituto Universitario de Educaci n F sica (2005). Gu a del diseo curricular. Colombia: Universidad de Antioquia, Instituto Universitario de Educaci n F sica.

8. Nieto, L. (2002). La flexibilidad curricular en la Educaci n Superior. Algunas perspectivas para su an lisis y ejemplos en la Educaci n Agron mica. En XXXII Reuni n Nacional de Directores de la Asociaci n Mexicana de Educaci n Agr cola Superior (AMEAS). Tuxtla Gut errez, Chis. $M$ xico. http://ambiental.uaslp.mx/docs/LMNC-PN-0210-FlexCurr.pdf 